\title{
Use of Probiotics as a Functional Food against Cancer
}

\author{
Abhinandan R Patil ${ }^{1 *}$, John I Disouza ${ }^{1}$ and Shivaji H Pawar ${ }^{1,2}$ \\ ${ }^{1}$ Centre for Interdisciplinary Research, D. Y. Patil University, Kolhapur, (MS), India \\ ${ }^{2}$ Centre for Innovative and Applied Research, Anekant Education Society, TCC Baramati, (MS), India \\ *Corresponding Author: Abhinandan Patil, Centre for Interdisciplinary Research, D. Y. Patil University, Kolhapur, (MS), India.
}

Received: July 10, 2019; Published: July 19, 2019

DOI: 10.31080/ASPS.2019.03.0347

Cancer is considered as the disorder of the cell in which the natural death (apoptotic) pathway of the cell cycle is lost resulting in uncontrolled growth [1]. The World health organization (WHO) has declared cancer as the second leading cause of death universally with a mortality rate of 9.5 million people's in only 2018. The report further says that 1 in 6 deaths reported globally is due to cancer, in which $70 \%$ of deaths are from low- and middleincome countries [2]. Colorectal is the leading third most cancer in which nearly 8, 62, 000 deaths were reported in 2018 [3]. To cope up with this problem, few new synthetic medicines are discovered after several years of clinical trials and many are discarded at the last phase of trials due to host-drug incompatibility issues. The treatments of colorectal cancer are mostly given by the systematic route rather than the local action at the site of the disorder. This results in the systematic toxicity and adverse effects on vital organs. So there is a need of the drug that can be delivered by peroral route with its local action, minimizing the systemic toxicity controlling morbidity and preventing the mortality [4].

The cost of these anticancer drugs and even certain antibiotics are beyond the reach of the common man and if made available are puzzled by the issue of innumerable side effects and suffering [5]. So, there is a need for such medicine which is from natural origin and deprived of its side effects. With the advancement in the studies of nutraceutical foods and the knowledge of nano-pharmacology, there is need of formulated functional food to heal gastrointestinal tract cancer [6]. This thing can achieved by deliver of the functional foods by the per-oral routes directly into gastrointestinal tract like food bolus. These nutraceuticals can act as a promising healer due to the natural origin and can be made available at a low price especially in developing countries like India.

Many holistic systems use the combinations of medicinal plants along with the fruits and vegetables containing the phytochemicals such as phenolic compounds, vitamins (vitamin A, C, and E) and fibers to prevent and treat cancer [7]. These component acts as functional food which along with nutrients provide the health benefits called nutraceuticals. Many green leafy vegetables and season fruits act as a functional food and provide health benefits. The many exotic nature of the medicinal plants is used by the tribal community to heal many kinds of bodily disorders since ancient times [8]. Polyphenols are the group which contains different medicinal component such as simple phenols, acetophenones, stilbenes, hydroxycinnamic acids, coumarins, lignans, phenolic acids, flavonoids, and tannins. These components are found effective in blocking the telomerase in cancer cell lines proved by many invitro and in-situ studies [9]. The probiotics are considered as the class of microorganism that are found as residential microflora of the gastrointestinal tract assisting the digestive and enzymatic function of the host [10]. Probiotics are the basic source of the functional food used as 'yogurt, dahi' but come with the short shelf life. Functional foods are used as the alternative source of healing component not morphological but by the physiological pathways. These are used as the therapeutical agent along with the diet to reduce the risks of various diseases as a prophylactic agent. The most common diseases that are treated by neutraceutical agents are gastrointestinal diseases and buccal cavity disorders. The pharmacological activities that are observed in case of probiotics are mostly by antioxidant mode of actions without the liver damage and carcinogenesis. The antioxidant mechanisms of probiotics could be due to metal ion chelation, enzyme inhibition and free radical scavenging mechanism. The synthetic drugs that are of the chemical origin or synthesized in the lab precipitate toxicity after long use.

The probiotics especially Lactobacillus genera are extensively explored for a long time as part of the diet. The origin of these microbes is mostly from the milk and its derived products. Since birth, these microbes work together as part of the digestive system 
as mutualism mode of adaption in the gastrointestinal tract. The probiotics fight the dysbiosis conditions with the pathogenic strains in the lumen of the intestine. In extreme condition, the inoculation of the Lactobacillus is found effective in suppressing and destroying the disease-causing microbes i.e. pathogens [11]. The probiotics include many different bacterial strains, each of these species differs in nature and activity. Most of the times strains of the same species are unique in characteristics such as adherence, immunological activity and other biological actions on the host. The major mechanism involved is the lowering of the $\mathrm{pH}$ in the colonic region, inhibiting the adhesion of the pathogenic strains to the intestinal lumen. Auto-aggregation and co-aggregation are the other two mechanisms that are inhibiting the colonization of pathogens. The hydrophobicity of the hostprobiotics interaction also prevents various diarrheal diseases [12-14]. Currently, probiotic research aims in the isolation of new strains and investigation of microbiota and microbiome characteristics in each part of the gastrointestinal tract from the small intestine to the colonic region. The basic focus of research includes the host-microbe interactions within the intestine, microbe-pathogen interactions within the microbiota and the microbe-drug interactions in diseased condition. The discovery of the new strains of Lactobacillus from the new natural source from the heavy crowd of the microbiota is a tricky and time-consuming process. The mechanism of action shown by the lactobacillus genera and it's sub-strains vary in its pharmacological activity. By the genotypic phylogeny study, all of Lactobacillus strains may fall under the same ancestral category but varies in the biochemical and physiological activities. This indicates that newly discovered strain of Lactobacillus may come with new pharmacological activity against different diseases. The whole genomic sequencing of newly discovered culture highlights the potential of the strain as a functional food in treating many dreadful disorders of the intestine. The positive interaction seen in all the above cases is mediated by means of the antagonistic activity of these probiotics.

Currently, in the market, there is a need for such nutraceutical products which may provide health benefits. Mostly the world is suffering from dreadful diseases like cancer. There are various types of cancer cases, in which colon cancer ranks third in India. The prophylaxis measures available for cancerous diseases are nearly zero as the etiology of the disease is unknown. However, the probiotics are proven useful in treating the diseases of the gastrointestinal tract such as diarrhoea and minor infection or manifestation by the pathogenic strains. Improper bowel habit is found responsible for causing the disease called adenomatous carcinoma a hybrid type of colorectal cancer. This gives the major clue that the Lactobacillus may be used as the major source to heal any kind of the disease including colonic cancer. Thus, probiotics especially Lactobacillus from different sources with formulation may act as functional nutraceutical mediators to heal diseases like cancer in future.

\section{Bibliography}

1. Patil AR., et al. "Shelf life stability of encapsulated lactic acid bacteria isolated from Sheep milk thrived in different milk as natural media". Small Ruminant Research 170 (2019): 19-25.

2. Patil AR., et al. "Lactobacillus Model Moiety a New Era Dosage Form as Nutraceuticals and Therapeutic Mediator". In Biotechnology and Bioforensics, SpringerBriefs in Applied Sciences and Technology. Springer, Singapore (2015): 11-21.

3. Lee H., et al. "Functional properties of Lactobacillus strains isolated from kimchi”. International Journal of Food Microbiology 145 (2011): 155-161.

4. Cuicui D., et al. "Reduction of aflatoxin B1 toxicity by Lactobacillus plantarum C88: a potential probiotic strain isolated from Chinese traditional fermented food-Tofu". PloS one 12 (2017): 1-16.

5. Patil AR., et al. "Probiotic potential of Lactobacillus plantarum with the cell adhesion properties". Journal of Global Pharma Technology 10 (2018): 1-6.

6. Jia., et al. "Diabetes Promotes DMH-Induced Colorectal Cancer by Increasing the Activity of Glycolytic Enzymes in Rats". PLOS ONE 9 (2014): e110455.

7. Amaretti A., et al. "Antioxidant properties of potentially probiotic bacteria: in vitro and in vivo activities". Applied Microbiology and Biotechnology 97 (2013): 809-817.

8. Balakrishnan., et al. "Antioxidant Activity of Coated Probiotic Lactobacillus casei on Chromium(VI) Induced Oxidative Stress in Rats". Proceedings of the National Academy of Sciences, India Section B: Biological Sciences 84 (2014): 305-310.

9. Christina G., et al. "Yogurt Consumption Does Not Enhance Immune Function in Healthy Premenopausal Women". Nutrition and Cancer 37 (2000): 27-35. 
10. Caroline B., et al. "Pretreatment with the probiotic VSL\#3 delays transition from inflammation to dysplasia in a rat model of colitis-associated cancer". American Journal of PhysiologyGastrointestinal and Liver Physiology 301 (2011): G1004G1013.

11. Fabien J., et al. "Milk Fermented by Propionibacterium freudenreichii Induces Apoptosis of HGT-1 Human Gastric Cancer Cells. Edited by Abbes Belkhiri". PLoS ONE 7 (2012): e31892.

12. Patil AR., et al. "Health benefits of probiotics by antioxidant activity: A review". Pharma times 50 (2018): 1-3.

13. Patil AR., et al. "Evaluation of Lactobacillus plantarum growth in milk of Indian buffalo breeds based on its physico-chemical content". Buffalo bulletin 38 (2019): 345-352.

Volume 3 Issue 8 August 2019

(c) All rights are reserved by Abhinandan R Patil., et al. 